\title{
A lighting study for air traffic control towers
}

\author{
S. Grignaffini \& M. Romagna \\ "Sapienza", University of Rome, Department of Astronautic, \\ Electric and Energetic Engineering (D.I.A.E.E.), Italy
}

\begin{abstract}
The purpose of this work was to determine the characteristics of glass and natural and artificial lighting systems capable of achieving, within the operating rooms of the air traffic control towers, the optimal conditions in terms of visual performance and comfort, within a framework established by law, from energy conservation, the availability of materials, ease of maintenance, security. The solutions identified, although the result of a compromise between different environmental and functional requirements often conflict with each other, were still subject to the need to ensure the proper and safe view of the external environment by air traffic controllers.

The methodology, given the complexity of the problems arising from the interaction between various parameters, is based on different and successive levels of detail, each of which is in preparation of the next. In particular, starting from a lumped parameter-analysis that considers only the global aspects of the problem and identifies the main characteristics of materials and systems used, is followed by a study of distributed parameter to analyze in detail the visual aspects and solutions possible, before arriving in the creation of design specifications whose validity has been verified by studying a case type. The different levels of analysis can be summarized as follows:
\end{abstract}

1. Lumped parameter analysis

2. Distributed parameter analysis: visual environment

3. Distributed parameter analysis: case study

Keywords: air traffic control towers, control room, natural lighting, artificial, lighting, glass, curtains, dimming, visual comfort. 


\section{Introduction}

The purpose of this work was to determine the characteristics of glass and natural and artificial lighting systems capable of achieving, within the operating rooms of the air traffic control towers, the optimal conditions in terms of visual performance and comfort, within a framework established by CIE (Commission Internationale de l'Eclairage) Standards [1-4], from energy conservation, the availability of materials, ease of maintenance, security. The solutions identified, although the result of a compromise between different environmental and functional requirements often conflict with each other, were still subject to the need to ensure the proper and safe view of the external environment by air traffic controllers.

The methodology, given the complexity of the problems arising from the interaction between various parameters, is based on different and successive levels of detail, each of which is in preparation of the next. In particular, starting from a lumped parameter-analysis that considers only the global aspects of the problem and identifies the main characteristics of materials and systems used, is followed by a study of distributed parameter to analyze in detail the visual aspects and solutions possible, before arriving in the creation of design specifications whose validity has been verified by studying a case type. The different levels of analysis can be summarized as follows:

- Lumped parameter analysis

The purpose of this first level is the identification of the main characteristics of the materials, particularly glass surfaces, to ensure both a low-energy and visual comfort (integrated analysis). The instrument used for this purpose is a kind of lumped-numerical simulation.

- Distributed parameter analysis: visual environment

The purpose of this second level is the identification of the main characteristics required to artificial lighting and natural in terms of performance and materials in order to obtain specific environmental and functional tower operations rooms in accordance with the regulations and based on information obtained from interviews, site inspections and measurements made in control towers of different importance and different sizes.

- Distributed parameter analysis: case study

The purpose of this third level is to evaluate in detail, on a real case, the results obtained on the basis of lighting design choices resulting from the first two levels, using commercial components. The type of material is further defined and specified, developed in the analysis than in lumped one. Different distributed parameter simulation packages were used, in order to reach the purpose applied to the geometry of the control room of a major Italian airport.

\section{Lumped parameter analysis}

The purpose of this section is to identify the optimal value which the following parameters must have: 
- Transparency and inclination of the glass surfaces

- Accommodation and transparency of internal screening components

- Type of lighting control system

The range of variability of these parameters within which to move to carry out the optimization, it appears to be rather limited as determined by common and established practice systems and some general rules related to the basic mode of operation of control rooms and on CIE Standards [5-7]. In particular, to reduce dazzle and reduce heat loss, the glasses are normally used transparencies in the visible range between $35 \%$ and $50 \%$ and shall be put in place with inclinations between $5^{\circ}$ and $30^{\circ}$, mainly to limit fouling, while the thermal transmittance range between 1.3 and $2\left[\mathrm{~W} / \mathrm{m}^{2} \mathrm{~K}\right]$ and are always accessible with the use of double glazing, and possibly with low-emissivity layer. Internal screening components consist of motorized blinds, controlled manually, which has transparency between $20 \%$ and $50 \%$. As for artificial lighting, the limit is represented by the European Standard EN 12464-1 [8], which provides an average illuminance of $500 \mathrm{~lx}$ dimmable; in particular, by an analysis of existing towers, reducing the flow of light is normally manually operated and this specific control is referred to the illumination of the working field, operated locally by each controller, while general lighting is centralized. The optimization is performed by using a numerical package, able to integrate aspects of thermal, natural and artificial lighting, the occupants' interaction with the systems of internal screening and control of the flux emitted by the lamps. The optimization parameter, once the visual and thermal requirements are guaranteed, is the total energy consumption.

The data used for the calculation are:

- latitude and external climatic conditions

- illumination of interior design

- design of the indoor temperature and humidity conditions.

The need to obtain results in terms of lighting and energy comparable with each other for the different Italian climatic conditions, imposed to preestablishing the size of the analyzed system, in order that all calculations refer to the same geometry.

\subsection{Methodological approach}

An analysis software, for the simulations, has been developed: it's a both of thermal and lighting energy integrated, consisting of a central thermal analysis interacting with sub modules that perform lighting calculations, simulations of control strategies and people interact with systems control. The program is based on the method of heat transfer functions TFM (Transfer Function Method), SATF (Air Space Transfer Function) in order to calculate the heat extracted and environmental temperatures. The module uses the lighting calculation program "Superlite" [9], which interacts with the program using its thermal calculations of solar radiation, solar elevation and azimuth. 
The meteorological data used as input of the simulations are the TMY (Typical Meteorological Year). The TMY hourly climate data are sequences most likely derived from calculations based on measurements made over two decades in major Italian cities using data from the stations of the Italian Air Force Weather Service; they report hourly data outside temperature, radiation direct sunlight and diffused in the horizontal plane, speed and wind direction, relative humidity, and were obtained by combining sequences of actual months. The simulations were performed for the city of Rome (latitude $42^{\circ} \mathrm{N}$ ), Milan (latitude $45^{\circ} \mathrm{N}$ ), Catania (latitude $38^{\circ} \mathrm{N}$ ). The change in the slope of the glass structure was planned in three steps, $0^{\circ}$ (angle of reference), $15^{\circ}$ (slope of the proposal) and $30^{\circ}$.The room is operational at any time throughout the day, with conditions of temperature in the range $20 \div 26^{\circ} \mathrm{C}$ depending on external environmental conditions. Interior heat load consists of the people, machines and lighting.

We considered two different commercial glazing systems:

- A double glazing which represents the average solution found during the inspections carried out in various Italian airports Control Towers. This system, shown in the simulations and diagrams with code EN1 is colored green/blue with a transmittance coefficient of 0.35 in the visible and thermal transmittance of $1.7 \mathrm{~W} /\left(\mathrm{m}^{2} \mathrm{~K}\right)$.

- A double glazing with outer pane constituted by a low emissivity and non-reflective deposit on surface 2: it has a "solar filter" behavior too. This system, shown in the simulations and diagrams with code EN2 is colored green, with a transmittance coefficient equal to 0.42 in the visible and thermal transmittance of $1.6 \mathrm{~W} /\left(\mathrm{m}^{2} \mathrm{~K}\right)$.

The control of natural light is obtained by closing motorized curtains operated directly by the operators. The management of the curtains has been suggested to be of linear type with adjustable phasing as a function of dazzle on the basis of site inspections carried out, the type of activity, the types of curtains used which severely limit or even prevent the external vision. In order to have best results, we analyzed the case in which the curtain is closed in the presence of dazzle (on/off) and the case in which the curtain is completely open; the second situation, based on studies carried out in offices, appears to be more likely in case of very light curtains, which allow both of the outside view and dazzle, produced by light scattered by the sky, protection. The same issues of simulation of human behavior occur in the management of the dimming of the lighting. In this case, since the actions are strongly influenced by the air traffic, we analyzed two opposite behaviors: completely off or turn on the light (on/off) dimming and on one or more areas.

\subsection{Energy and lighting analysis}

The results that have been reached are presented first to the analysis efficiency, and then in terms of lighting performance. The abbreviations used generally cover glasses (EN1, EN2, to indicate the two different types of glass referred to), the slope of the structure, which is explicitly mentioned in the angle of 
inclination to the vertical $\left(0^{\circ}, 15^{\circ}, 30^{\circ}\right)$, the type of regulation imposed on the system of artificial lighting ( $3 \mathrm{~d}, 2 \mathrm{~d}, 1 \mathrm{~d}, 0 \mathrm{~d}$ denote the setting or "dimming" to 3 , 2, 1 zone, or on/off), the adjustment of curtains (or index on/off, or to fully open or fully closed, L shows a linear setting), the type of curtain (as described above, $\mathrm{tA}, \mathrm{tB}, \mathrm{tC})$. The analysis of the results was conducted in steps, for each parameter, in order to reach some general conclusions, involving the variability of many parameters of interest for the study.

In order to evaluate the effect of different types of curtains, the use of glass EN2 was analyzed: since it's lighter than all'EN1, EN2 requires a more frequent use of the system of internal shading, and has a greater influence on energy demand. From this preliminary analysis, (Figure 1), it's possible to show that the choice of curtains is on average negligible in terms of energy, because it has a maximum variation of less than $4.5 \%$ in the worst case corresponding to the use a vertical glass and a light management of the on/off. The three curtains, substantially, lead to the same result as the energy behavior: so the different parameters will always refer to the curtain tA.

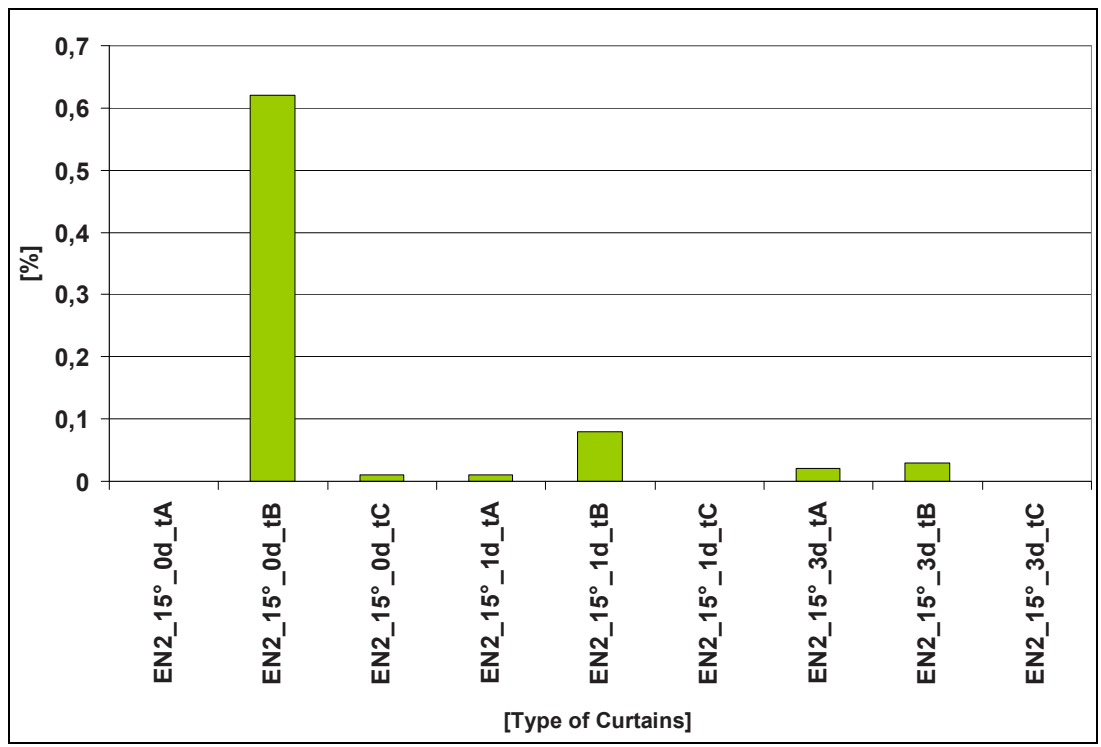

Figure 1: $\quad$ Rome case study: percentage change in consumption: glass EN2, linear adjustment of the curtains, structure with $15^{\circ}$ inclination and dimming 0,1 and 3 .

The influence of the inclination of the windows and the dimming are shown in Figure 2 for the glass EN1 and EN2.

From the results, it is clear that the use of more complex control of artificial lighting may be unnecessary, since the percentage differences in consumption are significantly small. 
Moreover, the use of a tilted transparent solution is on average, more efficient, if its slope is not excessive. The optimal solution is indeed to be angled $15^{\circ}$ from the vertical, as more fully shown in Figure 3 and Figure 4, respectively, with the glass and EN1 EN2. In particular, we can see that a simple increase from $15^{\circ}$ to $30^{\circ}$ can lead to increased consumption of up to $7.0-7.5 \%$ for glass EN2 and up to almost $8.0 \%$ for glass EN1. The influence of different types of dimming is very sensitive to the corner assembly, tending to fall in step vertical to the inclination of 30 degrees; in particular, we found maximum variations of $20 \%$ in the case of glass EN1 passing the case on/off dimming up to three zones.

The results obtained for the different energy behavior of the two windows considered, show that the structural solution at $15^{\circ}$ tilt is the best, regardless of the type of glass used. In this case, the glass EN2 is much better than the glass EN1 in terms of energy, especially if applied on a moderately sloped structure; moreover, the increase in complexity of the control system of artificial light from 1 to 3 dimming zones has little importance, since the improvement is around $0.8 \%$.

The overall representation of the various cases studied is shown in Figure 5, and defines the guidelines for purely energy choice of the best solutions for designing Control Tower environments in the climate of Rome.

The same type of analysis, developed for other latitudes, although with small percentage changes in consumption values obtained in different cases, confirms all that we reported for the climate of Rome.

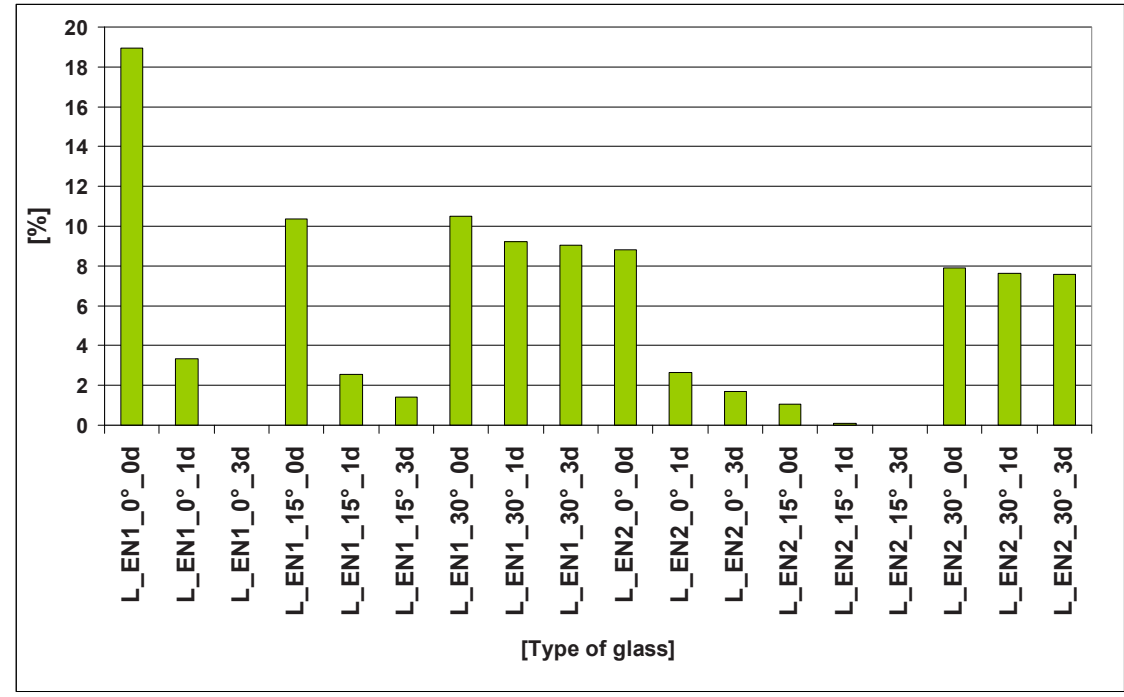

Figure 2: Rome case study: percentage change in consumption as a function of the control strategy of the lamps and the glasses (EN2 and EN1) linear adjustment of curtains. 


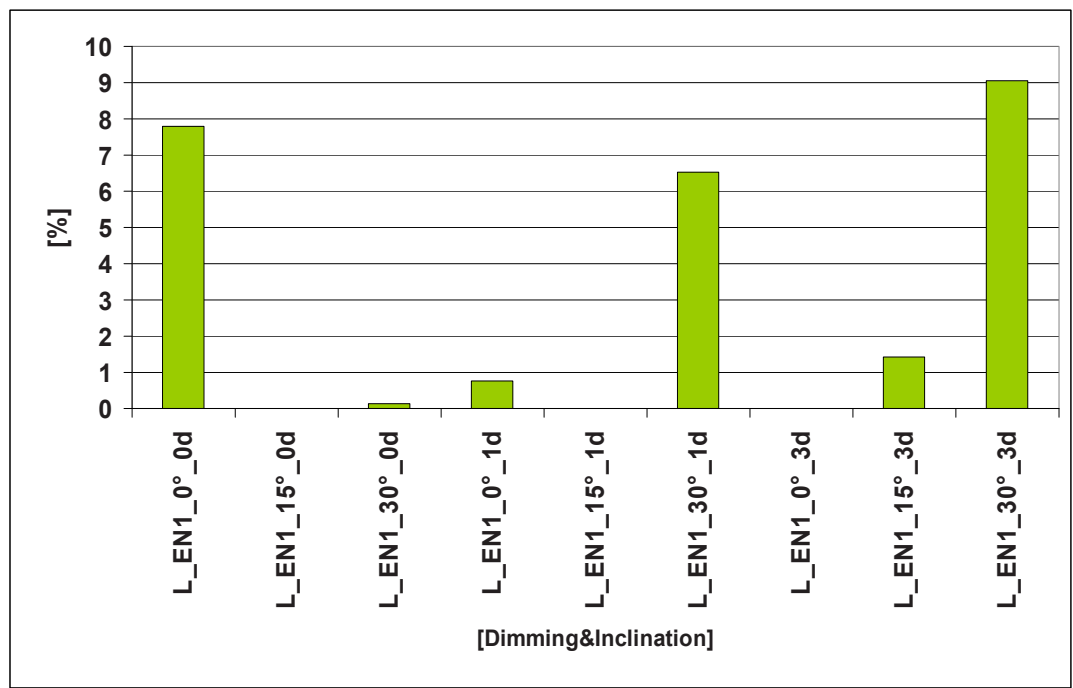

Figure 3: Rome case study: percentage change in consumption as a function of inclination and dimming; glass EN1.

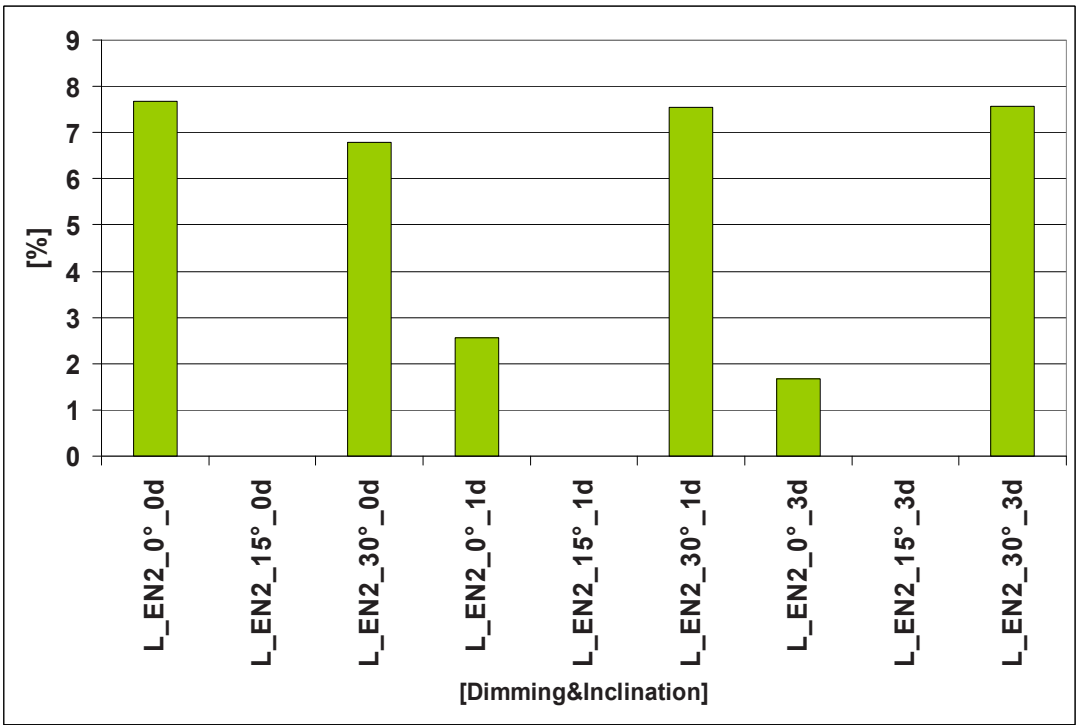

Figure 4: Rome case study: percentage change in consumption as a function of inclination and dimming; glass EN2. 


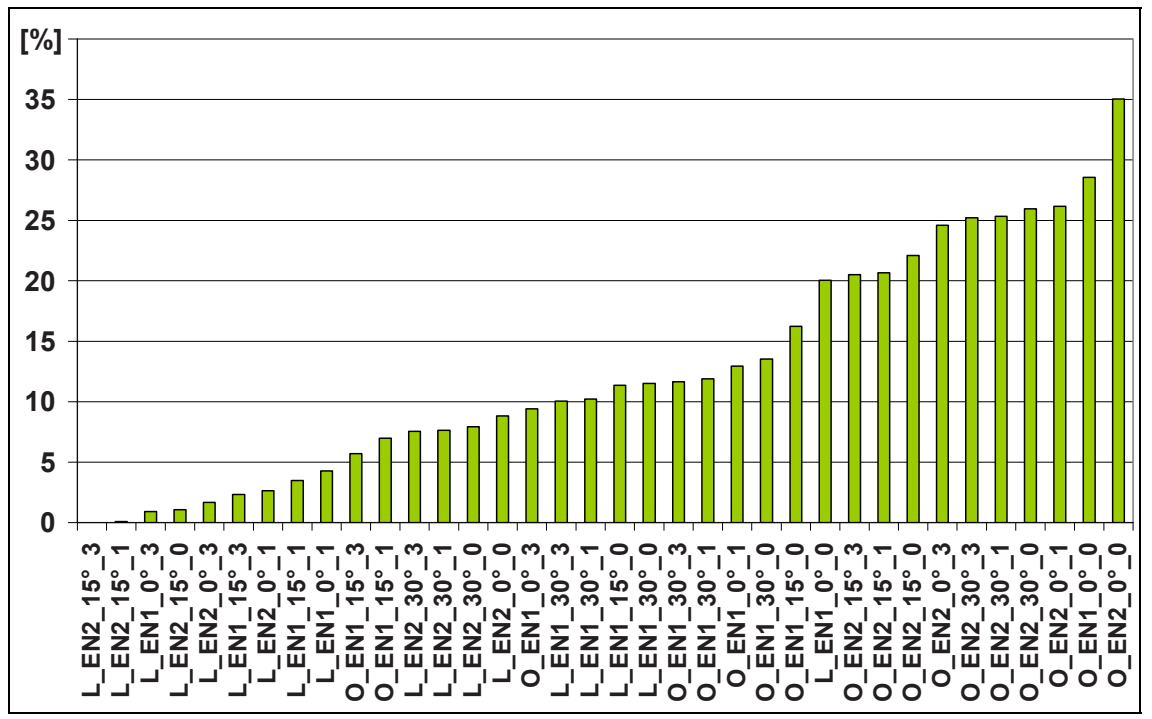

Figure 5: Rome case study: percentage change in consumption, overall analysis, and screen type tA.

\subsubsection{Lighting results}

This analysis deals primarily with natural light illumination; the annual distribution of illumination in the most disadvantaged point, generally and at the center of the room, has been studied for different control strategies and glass systems. In Figure 6, referring to the case of Rome, the annual cumulative distribution of natural light is shown; in other words it's the percentage of working hours in the period during which natural light is greater than a given value in the less illuminated environment. In Figure 14 we can observe that all control systems have an acceptable behavior, since they ensure a minimum of $500 \mathrm{~lx}$ for $30 \%$ of working hours throughout the day, including even the night. Various curtains have essentially the same behavior in terms of cumulative distribution, even if they differ in terms of protection from dazzle. The system EN1 shows a cumulative distribution percentage of $27 \%$ at $500 \mathrm{~lx}$, with a structure $30^{\circ}$ tilted, $35.5 \%$ for inclination of $15^{\circ}$, and $38 \%$ with a vertical structure; instead for the system EN2 these values become respectively about $30 \%, 37 \%$ and $41 \%$. Due to its higher transmittance, glass EN2 shows increasing values of cumulative distribution than the glass EN1, and it's preferable both in terms of lighting and energy, because it reduces the number of hours that may become necessary for the use of artificial lighting to ensure the required value of lighting design. The inclination is very important in terms of lighting, and even in this case the inclination of $15^{\circ}$ is much more preferable than a higher one, with differences of small percentage, which in this case are be very significant. 


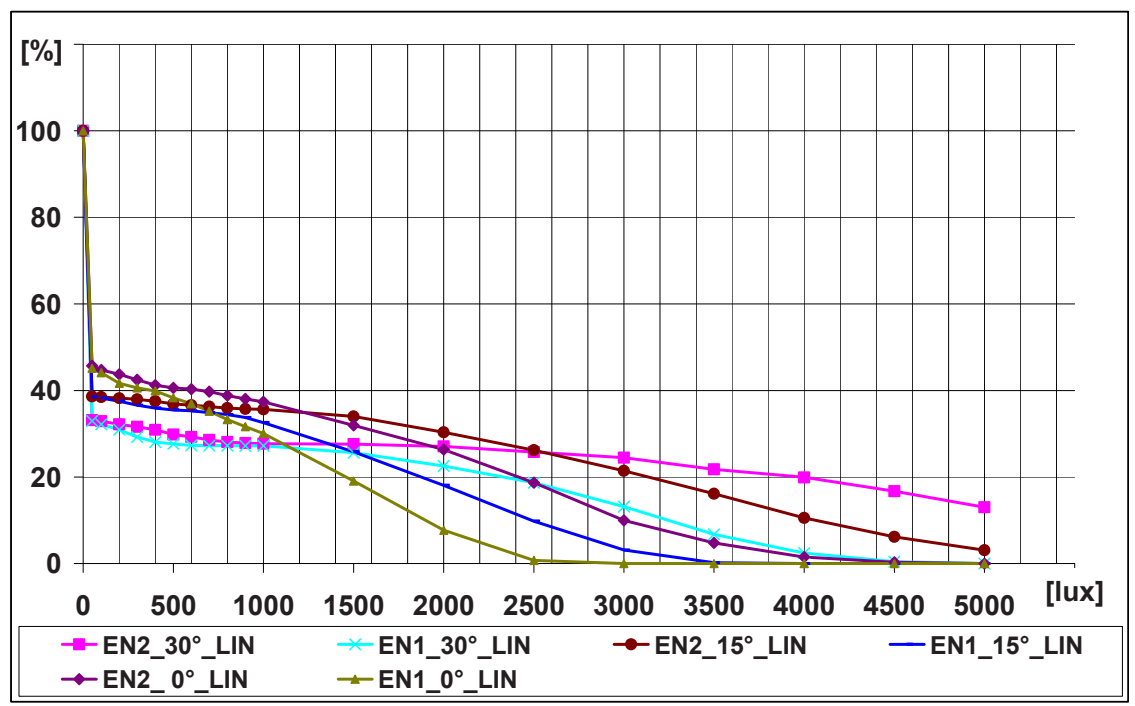

Figure 6: Rome case study: cumulative annual distribution of natural lighting: overall results, adjustment linear strategy of the curtains and screen $\mathrm{tA}$.

Another interesting aspect is related to the internal behavior of the curtain: EN2, with greater visual transmittance, is characterized by a minor number of hours with light on, but also, for the same reason, the maximum number of hours with curtains closed, resulting in continuous shielding from the external environment and risk of exclusion from the outside for occupants.

\subsection{Lumped Parameter Analysis: conclusions}

The lighting and energy analysis were carried out independently, to show the characteristics of the different alternatives simulated. So it is clear that the best solutions are those with the most efficient in both performance testing. Based on the analysis carried out we can observe that:

- The glazing system must have a light transmittance greater than $35 \%$, a low solar transmittance, less than $30 \%$, and a low reflection coefficient (less than 11\%);

- The glazing system must have an inclination between $12^{\circ}$ and $15^{\circ}$ from the vertical;

- the internal screen must have filtering capabilities to reduce dazzle and reduce energy consumption, against the direct and diffuse radiation; in particular the latter two functions may be delegated to two different curtains: the first, in face of the environment, which allows the vision of external objects, must have control function of the dazzle from diffused light, and the second one for direct light; 
- the installation of artificial lighting must be divided into zones and/or into dimming system that, depending on the activities, should be devoted to:

- $\quad$ general lighting

- $\quad$ lighting directed on the work area (task area)

- transit lighting.

\section{Distributed parameter analysis}

The purpose of this part of the work is an accurate and detailed characterization of the issues related to natural and artificial lighting, in order to achieve a detailed definition of the performance requirements for systems and equipment. The tools at the base of the reached solutions are the use of the results obtained by the interviews with operators, the legislation and the technical and commercial aspects of the available materials. In particular, the goals of this analysis are:

- ensuring occupants an environment where the vision is efficient, accurate and precise as suggested by the specific regulations;

- ensuring occupants an environment where the vision is easy and comfortable; this means that the efficient realization of a vision should not cause discomfort, ensuring an appropriate degree of visual comfort;

- $\quad$ ensuring the proper integration of environmental impact of artificial lighting, which is optimal in terms of visual comfort and energy savings, by an appropriate choice of elements and strategies of regulation and control;

- $\quad$ safety, flexibility, durability, low cost of the system.

\subsection{Visual environment}

In the study of the visual environment, two different phases, having different objectives, can be identified.

The first phase, whose purpose is to provide the most detailed knowledge of the features of the control room lighting, is based on the survey and measurement of the main lighting and photometric characteristics of the situation in the different air traffic control tower.

The second phase takes into account the fact that the feelings more or less positive that you can try "living" environment, however, depend on the impressions of physical, physiological and psychological environment that creates in users. For this purpose a questionnaire was constructed as a way of investigation to better know the expectations of the users, air traffic controllers, and to evaluate the level of satisfaction of individual needs. This instrument, even if delicate and of difficult interpretation, allows to face the problem of vision globally. The environment of air traffic control tower, although in some aspects can be compared to an office for the presence of computers, has its own peculiar needs of visual contact with the external environment by the use of glass walls exclusively. 


\subsubsection{TWR control room lighting}

Most of the information, in tower control rooms, is visual and affects primarily reading, handwriting and/or by computer, work with display screen and a visual check of the aircraft. So for a good vision it's important to have:

- suitable light source;

- uniformity of illumination;

- limitation of direct dazzle produced by light sources within the field of vision, and dazzle caused by the specular reflection of one or more objects that receive light from sources inside or outside;

- optimal color rendering;

- static and dynamic balance of luminance;

- control of the shadows;

- integration of natural and artificial light.

The EN 12464-1 provides an environment specifically for control towers, a value of average illuminance on the work plane of $500 \mathrm{~lx}$, with reference to the average value of exercise guaranteed by the installation of artificial lighting, and that is the average state degradation of the system; the CIE (Commission Internationale de l'Eclairage) values are in agreement with these proposed regulations. The regulations also require that artificial lighting is adjustable, that the quality class for dazzle reduction is high, that the environment is considered as the type of environment with the visual task very difficult (for the standard EN 12464-1 UGR $\leq 16$ ), that the color rendering index is $\mathrm{Ra} \geq 80$, that natural light dazzle is avoided, that duplication of reflections on windows and displays, especially in night time are avoided and that the reflected dazzle on the monitor is limited with luminance of furniture and reflex areas less than $200 \mathrm{~cd} / \mathrm{m}^{2}$. The investigations in the field have shown that operators use mainly during the night table lamps, with average illumination of $300 \mathrm{~lx}$ to avoid excessive contrast between interior and exterior environment. Regard to the illuminance values in the daytime period, the mostly transparent structure ensures values high enough and the assistance or intervention of artificial light is not required, except in periods of transition or, in special cases, to balance luminance contrasts. The situation is quite different for the problems relating to natural light, where there are no regulation advices, if not aimed at maximizing its use, clearly for reasons of energy conservation, while limiting the situations of discomfort caused by direct illumination and reflection caused by the same natural light. Another important aspect is the proper distribution of luminance in the field of view, taking into account the fact that operator activity is not conducted exclusively in a static way, that the operator doesn't focus on a particular plane and his activity takes place under dynamic conditions. The luminance values in the environment must be balanced as far as possible, in order to not have excessive contrasts in the direction of normal observation of the operators. To get a good visual performance, it is important to create a just and balanced distribution of luminance, but is not necessary the actual uniformity of the same. The human eye is able to adapt to an intermediate level between the luminance of the visual task and the surrounding areas, as a function of luminance, size, and position on the various areas within the visual field; as the greater the difference between 
these luminances, the more difficult the eye will be able to adapt to the environment, creating situations that can come to lead, in extreme cases, uncomforted situations. Everything is still valid and effective until it considers an artificial lighting system; it's a different matter when analyzing the problem from the natural light point of view, which inevitably creates differences difficult to manage, and is affected by unpredictable variations. Dazzle is mainly caused by excessive luminance values and luminance contrasts too high. For air traffic control tower it's quite difficult to eliminate the cause of dazzle produced by excessive natural luminance and luminance contrast, because the need to maintain a constant visual check with the outside prevents an appropriate and effective screening of the glass surfaces. Anyway it's possible to take lower transparency of transparent surfaces, compatibly with the energy introduced by this solution, and the shielding efficiency, possibly perforated, to allow effective screening and the ability to ensure the vision. In such work environment that we suggest a shade of neutral and warm light, as proposed by the regulations.

\section{References}

[1] CIE, 1984. Publication CIE 60-1984: Vision and the Visual Display Unit Work Station, Commission Internationale de l'Eclairage, ISBN: 9789637251078.

[2] CIE, 1983. Publication CIE 55-1983: Discomfort Glare in the Interior Working Environment, Commission Internationale de l'Eclairage, ISBN: 9789290340553.

[3] CIE, 1995. Publication CIE 117-1995: Discomfort Glare in Interior Lighting, Commission Internationale de l'Eclairage , ISBN: 9783900734701.

[4] CIE, 1997. Publication CIE 23-1997: Low vision - Lighting Needs for the Partially Sighted, Commission Internationale de l'Eclairage, ISBN: 9783900734787.

[5] CIE, 1982. Publication CIE 52-1982: Calculations for Interior Lighting Applied Method, Commission Internationale de l'Eclairage, ISBN: 9789290340522.

[6] EN 15193, 2007. European Standard EN 15193:2007 Energy performance of buildings - Energy requirements for lighting.

[7] CIE, 1986. Publication CIE 29.2-1986: Guide on Interior Lighting, Commission Internationale de l'Eclairage.

[8] EN 12464-1, 2002. European Standard EN 12464-1:2002 Light and lighting- lighting of work places- Part 1: Indoor Work Places.

[9] Building Technologies Department - Ernest Orlando Lawrence Berkeley National Laboratory, 1 Cyclotron Road, MS 90-3111Berkeley, California 94720, http://btech.lbl.gov. 